\section{Enhanced Spontaneous Anti-Tumor Cytotoxicity of Natural Killer Cells in Cutaneous T-Cell Lymphoma Patients}

\section{Abstract} Cutaneous T-cell Iymphoma (CTCL) is characterized by the expansion of malignant CD4+ T
cells in the skin. There are two main subtypes of CTCL, mycosis fungoides (MF) and Sézary
syndrome (SS). Previous studies have demonstrated defects in cell-mediated immunity. including altered cytokine profiles and decreased neutrophil function, and patients often have recurring bacterial and viral infections. Recent studies have shown increased expression of Interleukin (IL)-15 in malignant CD4+ T cells in CTCL patients (Mishra et al., Cancer Discovery, 2016). Since IL-15 can enhance NK cell differentiation and activation, we hypothesized that NK
cells from CTCL patients might have phenotypic and functional alterations due to these changes in homeostatic LL-15 levels.

To evaluate the absolute number of NK cells in CTCL patients, CD56+Lineage- lymphocytes were quantitated by flow cytometry. SS patients had significantly fewer NK cells as compared to normal donors (mean $\pm S E M$ of absolute cell numbers in normal donors $=0.2442 \pm 0.02, n=51$
$S S=0.1072 \pm 0.02, n=9$; $M F=0.2161 \pm 0.01, n=112$; unpaired $t$-test normal vs. $S S, p=0.007$ ) $\mathrm{NK}$ cell counts were significantly associated with overall survival, with the short-term risk of death increasing by $87.5 \%$ for every increase in 0.1 of absolute NK cell counts on univariate analysis utilizing the Cox proportional hazards model (Figure $1 A ; p=0.0413)$. We then examined
the cytotoxic capacity of $N K$ cells (CD56+Lineage-) freshly purified from both MF and $S S$ patients by standard chromium release assay. There was a significantly higher level of specific (to norma $=27.47 \pm 4.76, n=13$ vs. $52.54 \pm 4.88, n=12$, unpaired $t$-test, $p=0.001$ ). Upon further transcrip analysis of NK cell cytolytic mediators srom CTCL patients compared to normal donors, we
and $C T C L=91.39 \pm 9.18, n=3$ vs. $485.7 \pm 43.15, n=7$, unpaired $\mathrm{t}$-test, $\mathrm{p}=0.0004$ ) and granzyme increased levels of insterferon-g $38.13, n=7$ unpaired t-test, $p=0.03$ ). In addition, we observe $n=7$; unpaired $t$-test $p=0.007$, indicating NK cells from CTCL patients exhibited consistently expression pattern of the IL-15 receptor complex on NK cells in CTCL patients as compared to normal donors by RNA sequencing. There was a significant elevation in IL-15Ra (mean \pm SEM and IL-15RY (57.02 $\pm 7.525, n=3$ vs. $154.7 \pm 12.14, n=7 ; p=0.001)$, while IL-15R $\beta$ was also elevated (128 $\pm 23.68, n=3$ vs. $223.2 \pm 25.43, n=7 ; p=0.056$ ). Next we evaluated protein levels of phosphorylated signal transducer and activator of transcription 5 (p-STAT5) in fresh whole in p-STAT5 in CTCL patients as compared to normal donors (mean \pm SEM of fluorescent
in nsity in normal donters

These data suggest that NK cell activation is enhanced in patients with CTCL, including MF patients wh disease locelyed

\section{Results}

Figure 1. NK cell numbers are associated with overall survival in CTCL

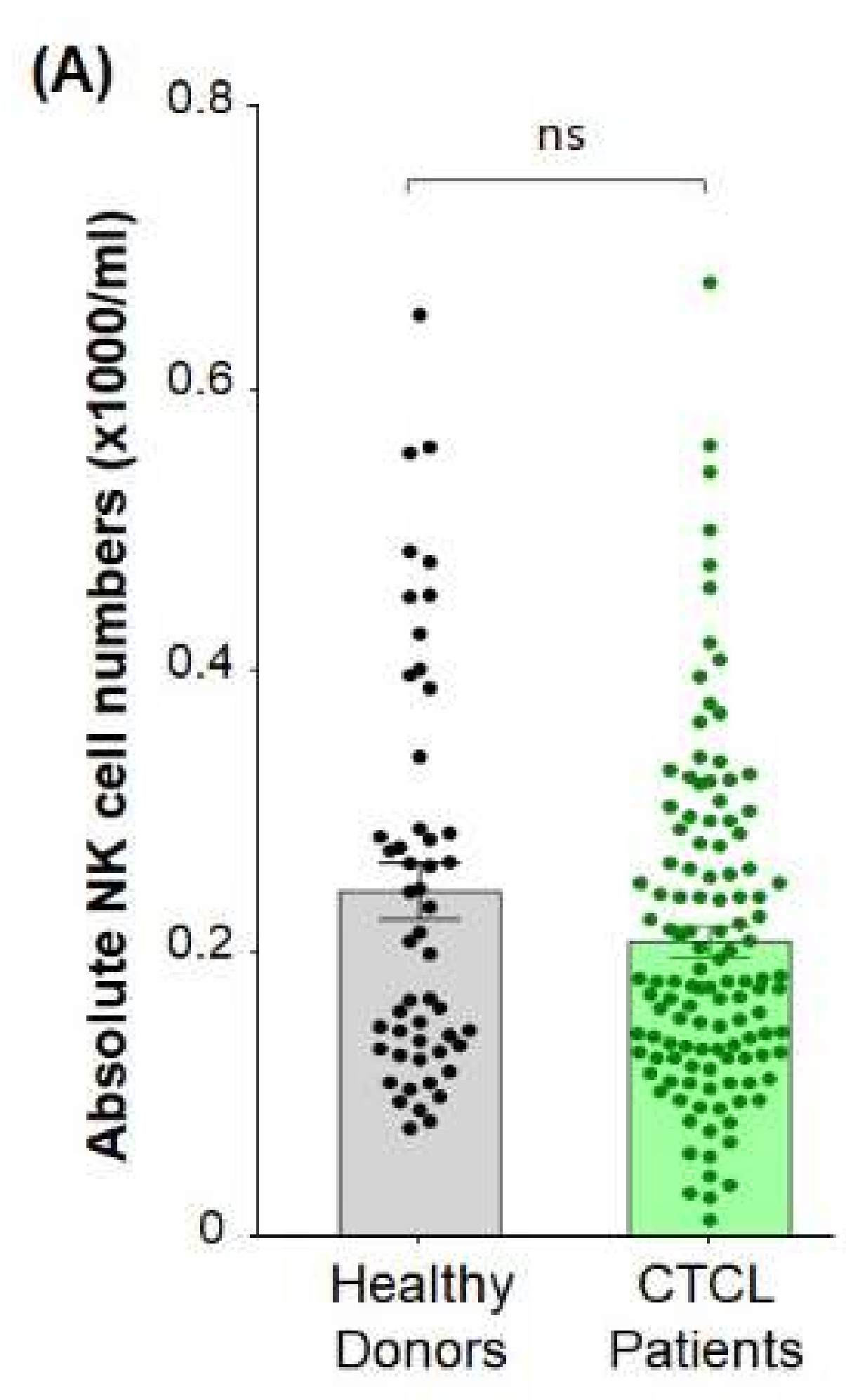

(B) Cox proportional hazards regression

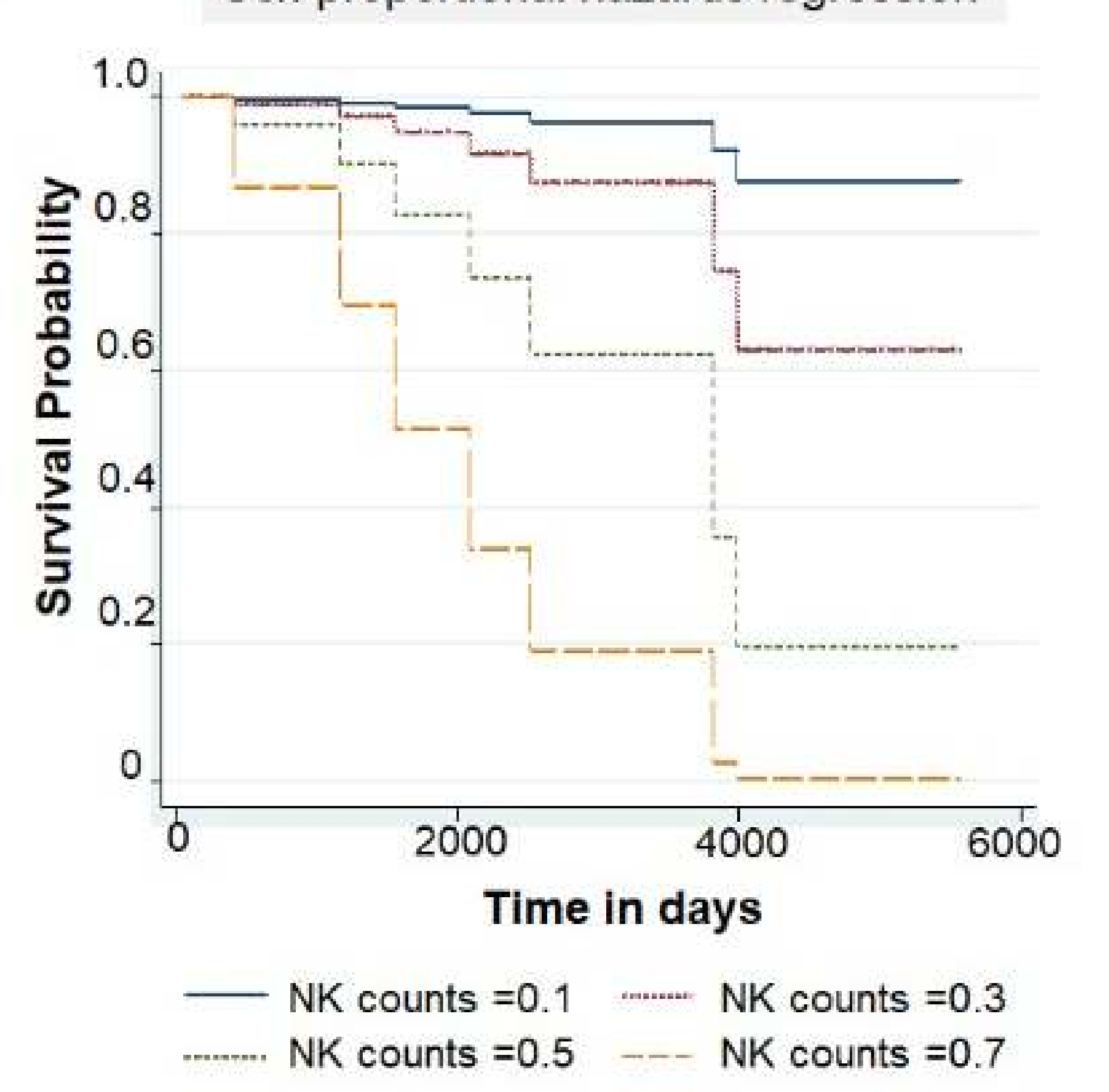

Figure 1. (A) The absolute number of NK cells in CTCL patients and healthy normal donors was determined by flow cytometry
association between absolute NK cell counts and oveagll survival of early nend late stage CTCL
patients. Interestingly. NK cell counts were significantly associated with overall survival, with the patients. Interestingly, NK cell counts were significantly associated with overall survival, with the
short-term risk of death increasing by $87.5 \%$ for every increase in 0.1 of absolute NK cell counts on

\title{
Results (cont.)
}

Figure 2. NK cells from CTCL patients exhibit increased cytotoxicity against tumor targets ex vivo

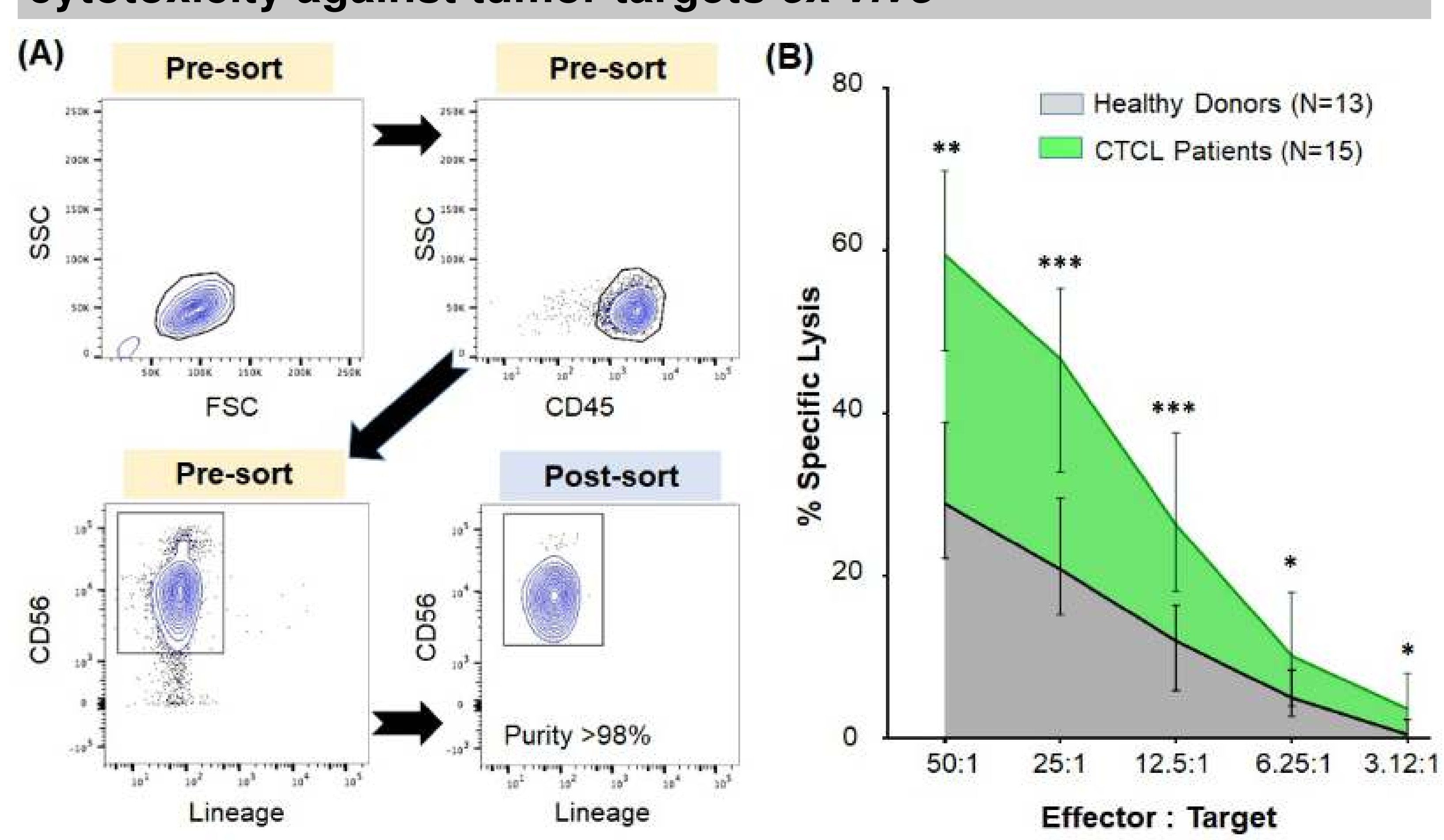

Figure 2. NK cells from normal healthy donors or CTCL patients were isolated from peripheral
bood and sorted to purity. (B) NK cell function was assessed by standard chromium release assay blood and sorted to purity. (B) NK cell function was assessed by standard chromium release assay,
using purified NK cells as effectors cells at the ratio indicated and K562 cells as the tumor targets.

Figure 3. NK cells from CTCL patients display activated transcriptional profile compared to healthy donors

(A)

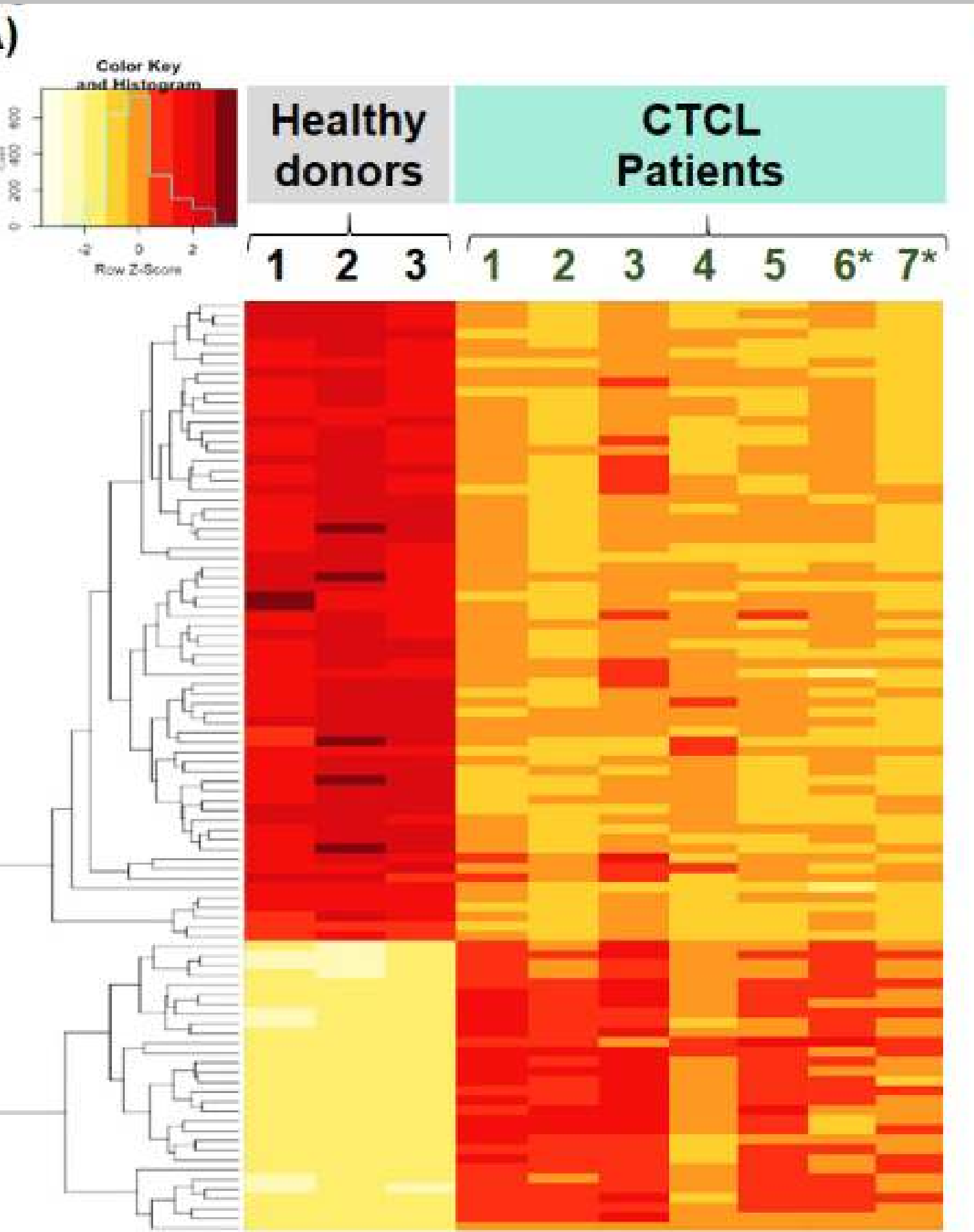
Figure 3. NK cells were isolated from CTCL patients and normal donors and evaluated by RNA
sequencing. (A) Heatmap of differentially regulated transcripts between normal and CTCL patients
(n=5 MF, $n=2 \mathrm{SS}^{*}$, and $n=3$ normal donors).
(B) Mediators of cytolytic activity and NK cell as compared to normal controls $(n=3)$. Figure 4. Ingenuity pathway analysis of NK cells in CTCL

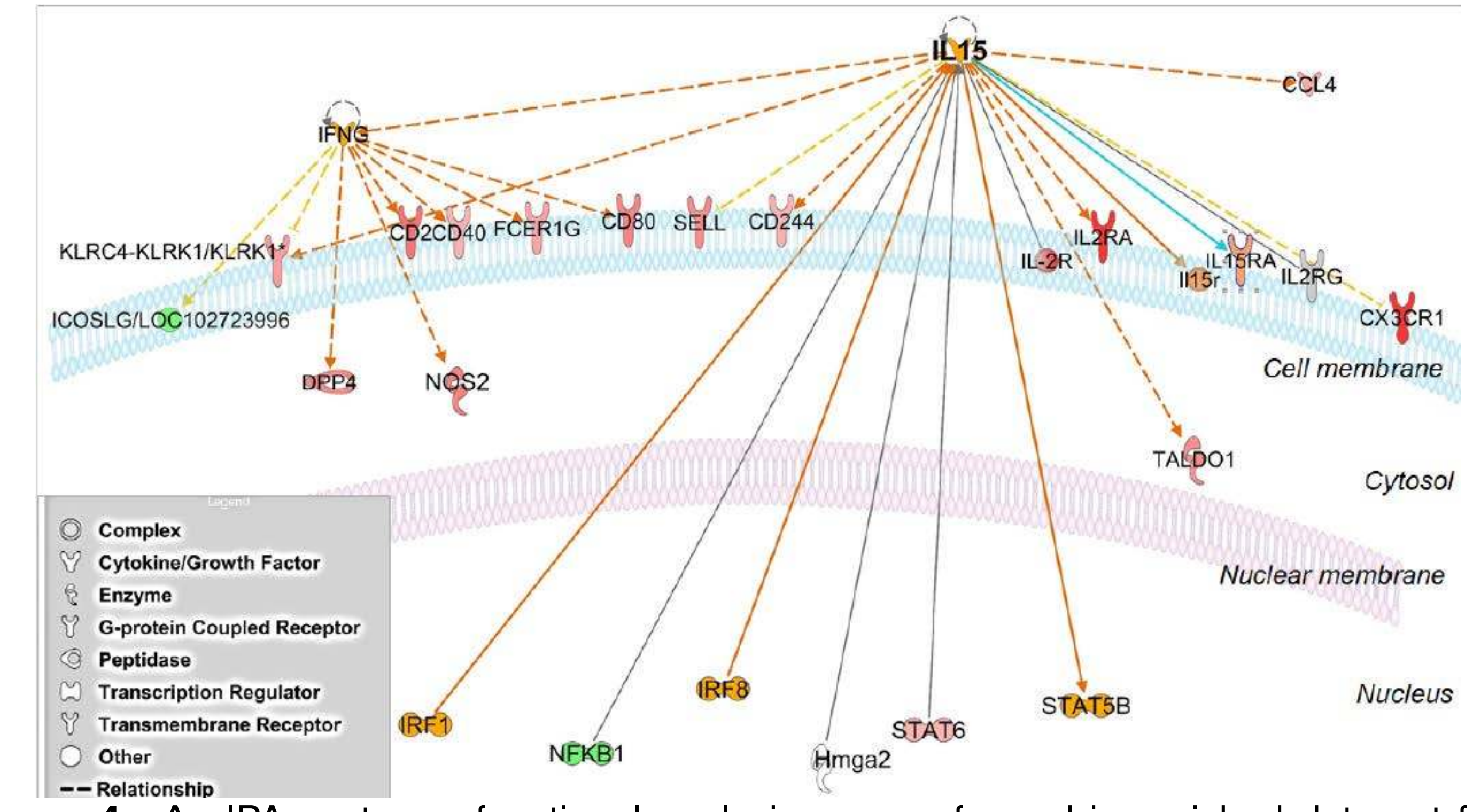

Figure 4 . Antostip
cells. Red ind upstream functional analysis was performed in enriched data set from the NK
ingregulation in CTCL patients as compared to normal donors, while green

\section{Results (cont.)}

Figure 5. IL-2 and IL-15 signaling pathways are altered in CTCL patients.

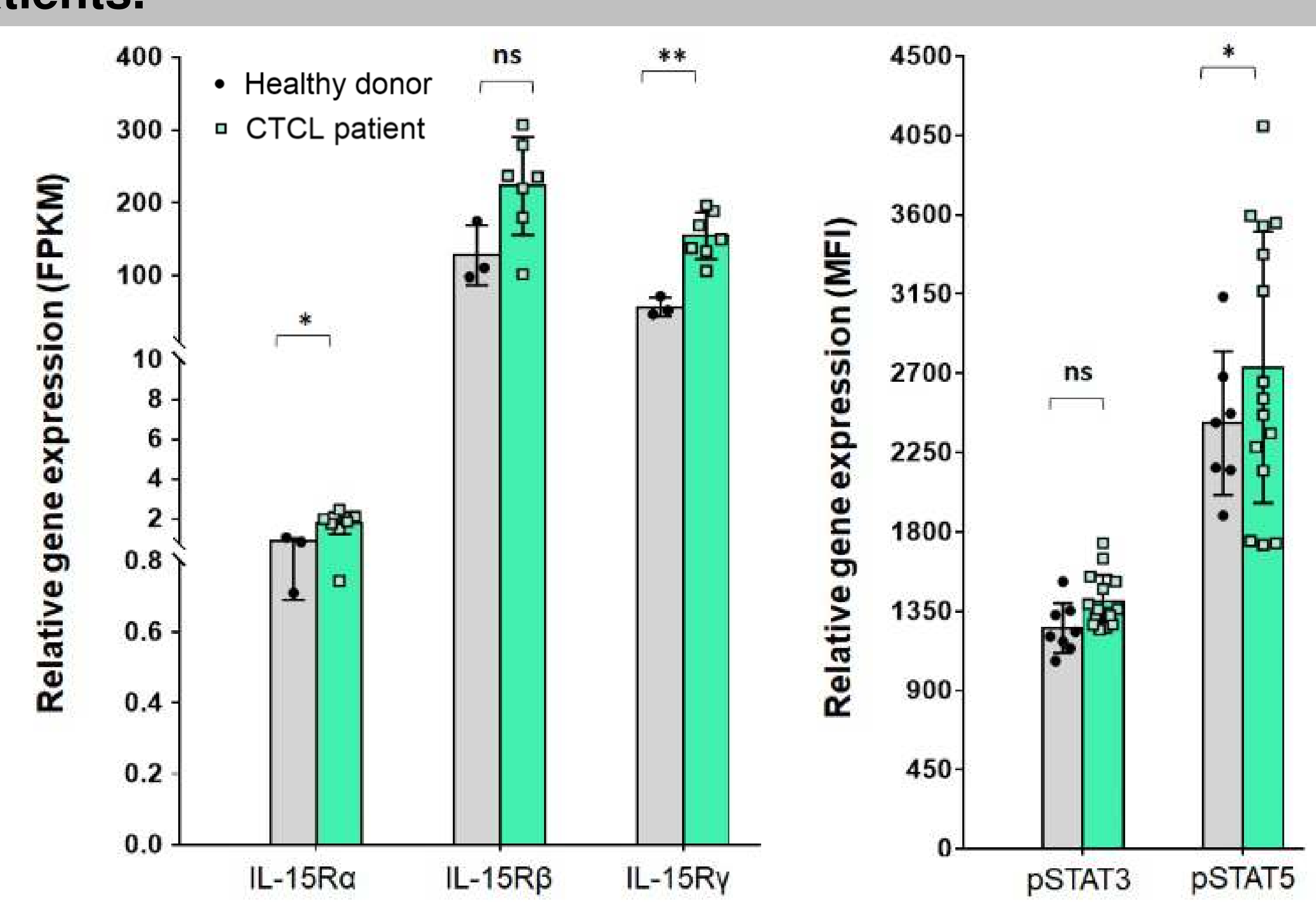

Figure 5. Purified NK cells were evaluated by RNA sequencing analysis ( $n=7$ CTCL vs. $n=3$ normal
donors). (A) RNA expression of receptor components required for both IL-2 and IL-15 signaling were evaluated in CTCL patients and compared with normal donors. (B) Protein expression of
phosphorylated STAT3 (pSTAT3) and STAT5 (pSTAT5) on NK cells was determined in freshly phosphorylated STAT3 (PSTAT3) and STAT5 (PSTAT5) on NK cells was determined in freshly
obtained whole blood samples from CTCL patients and matched normal donors by flow cytometry.
Graph indicates median fluorescent intensity (MFI) of normal (gray bars, $n=7$ ) as compared to Graph indicates median fluoresce
CTCL patients (green bars, $n=15$ ).

Figure 6. CD4+ malignant cells produce IL-15 which may lead to enhanced NK cell activation and function.

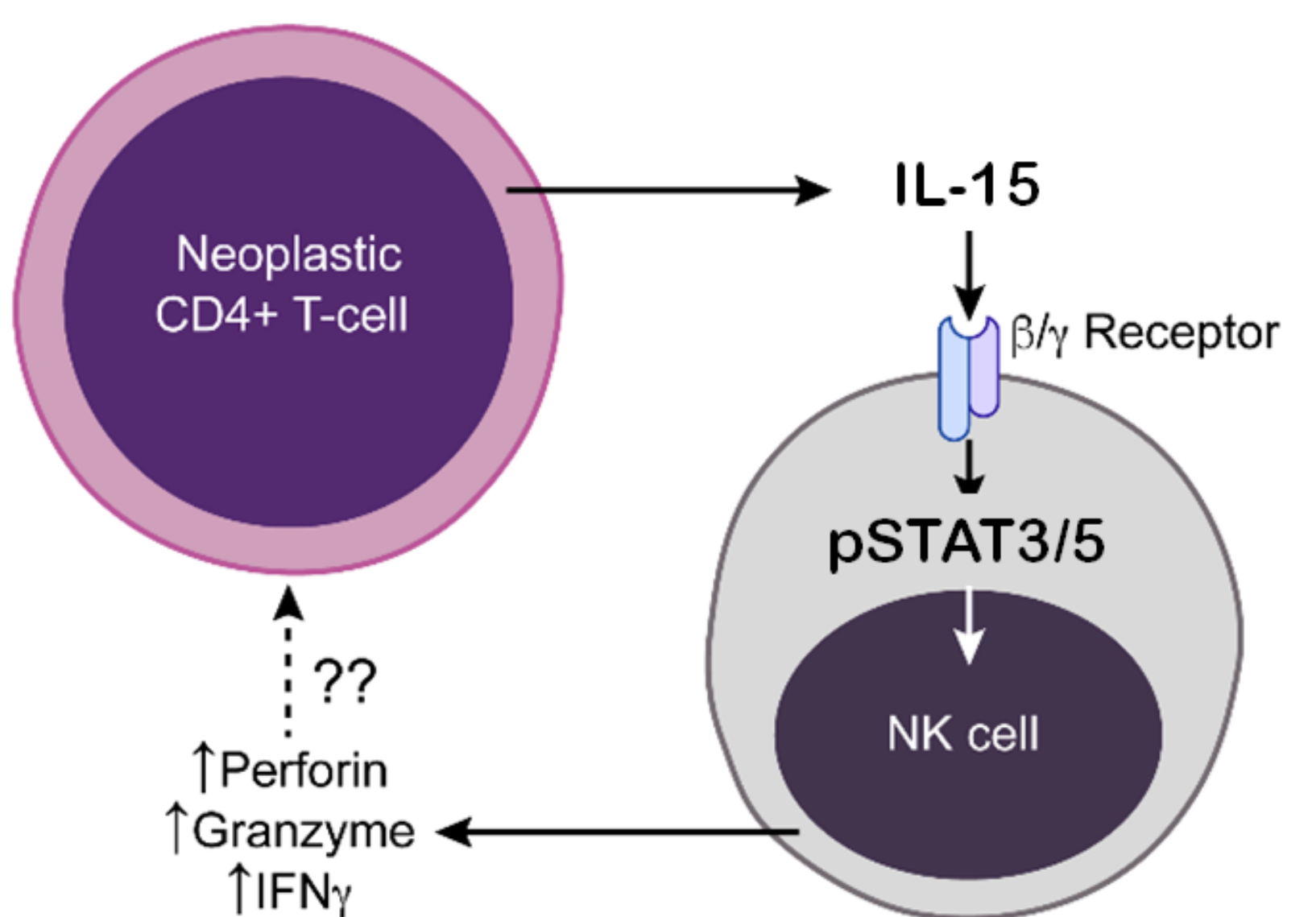

Figure 6. Schematic of interaction between malignant $C D 4+T$ cells in $C T C L$ patients producing IL15, which binds to the upregul
activating pathways in NK cells.

\section{Conclusions}

Conclusions. While our initial goal with this work was to define the functional capacity of freshly isolated NK. cells in CTCL patients, our comprehensive analysis of both NK cell function and
expression profiles uncovered intriguing results. One of the most interesting findings of this study expression profiles uncovered intriguing results. One of the most interesting findings of this study
was the significant association betteen NK cell number and CTCL patient survival. Similar to
previous reports, we find significantly decreased NK cell numbers in levkemic CTCL patients when previous reports, we find significantly decreased NK cell numbers in leukemic CTCL patients when
compared to healthy donors, but to our knowledge this is the first description of higher NK cell numbers in a malignancy being associated with decreased short-erm survivability. The cause
this significant clinical relationship is unknown. Few recent studies have described the tumorpromoting potential of NK cells through their ability to upregulate certain oncogenic pathways such
as VEGF-A, an activity mediated in part by reduced STAT5 activity. Notably, we describe increased pSTAT5 and did not identify upregulation of VEGF-A or other common tumor-promoting factors in our RNA sequencing analysis of NK cells from CTCL patients. In addition, we did not see patients. It is possible that there are other yet-undiscovered mechanisms of tumor promotion by
NK cells, which may respond to the tumor microenvironment or specific signals from tumor cells hemselves. These mechanisms may lead to failure of $\mathrm{NK}$ cells to control tumor progression recognition is warranted, however it is clear the overall immunosuppressive microenvironment in progression. Acknowledgements. BLM-B is funded by the American Association for Cancer Research (17-20-
46-MUND). Funding support was also provided by the Spatz Foundation (AMM), American Skin

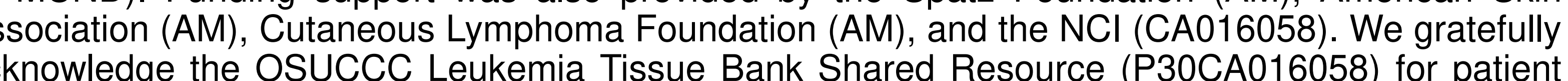
acknowledge the OSUCCC Leukemia Tissue Bank Shared
samples. No authors have any conflicts of interest to disclose 